\title{
Endoscopic optic nerve decompression for direct traumatic optic neuropathy: our 10 years experience
}

\author{
Vivek Sasindran ${ }^{1}$ and Mithra John ${ }^{1}$ \\ ${ }^{1}$ Pushpagiri Medical College Hospital
}

June 17, 2020

\begin{abstract}
Background: Traumatic optic neuropathy (TON) can be caused by direct or indirect injury. Direct optic injury usually results from optic nerve avulsion or laceration, or from direct fracture of the optic canal. Indirect optic injury is caused by increased intracanalicular pressure after an injury. The prognosis of direct optic injury is usually quite poor. To date, no standardized treatment protocol has been developed for TON. In this study we are assessing the visual improvement in patients with direct TON who underwent endoscopic optic nerve decompression in the last 10 years. Methods: 32 cases of optic nerve decompression for direct TON were done in the last 10 years. Preoperative and post operative visual assessment were done and followed up for 3 months, Results: There was complete improvement in vision in $59 \%$ of patients when optic nerve decompression was done within 72 hours of trauma; whereas $31 \%$ cases had partial improvement when done between 3 and 7 days. And there was $0 \%$ improvement when done after 7 days. Conclusion: Endoscopic optic nerve decompression is a minimally invasive effective method in direct traumatic optic neuropathy with minimal or no complications when done by well experienced ENT surgeon with ample knowledge of anatomy and anatomical variations. Other important prognostic factors include timing of surgery, extend of trauma and preoperative visual status Key words: Endoscopic optic nerve decompression (EOND), Nerve sheah incision/ fenestration, Traumatic optic neuropathy (TON), Visual assessment( VA), Visual improvement scale (VIS)
\end{abstract}

\section{INTRODUCTION}

Traumatic optic neuropathy (TON) can occur along any level of the optic nerve and results in partial to complete loss of visual acuity. TON can be classified into direct and indirect subtypes. Direct TON results from fractures involving the orbit or optic canal such as those associated with projectiles, facial smashes, or iatrogenic during endoscopic sinus surgery. Conversely, forces transmitted at different levels to the optic nerve after blunt head trauma can cause indirect TON. The damage to the optic nerve is hypothesized to be secondary to intraneural edema, hematoma, shearing of nerve fibers, altered cerebrospinal fluid circulation, and impaired retroaxonal transport of brain-derived growth factors ${ }^{1-3}$. It occurs in about $2.5 \%$ of midface fractures and $10 \%$ of craniofacial fractures ${ }^{4-5}$. For decades, treatment of compressive optic nerve neuropathy was conservative, with intravenous application of high dose corticoids, and in later years in combination with nerve growth factors ${ }^{6,7}$. Surgical treatment for traumatic optic neuropathy was not routinely done in the past as a minimally invasive approach to the optic nerve was not available; also the available intracranial or pterional approaches could add to the morbidity of the patient. However with advent of endoscopic sinus surgeries, a new corridor for optic nerve decompression started to open up. The treatment of TON is controversial; primarily because of absence of a standard treatment protocol- medical as well as surgical. Secondly spontaneous improvement of vision in patients with TON has been reported in 20-38\% of untreated cases ${ }^{8,9}$. The aim of EOND is to decompress the bony optic nerve canal along with removal of bony fragments with or without impingement on the optic nerve as well as reducing the intracanalicular pressure 
by added fenestration of nerve sheath in selected patients; expecting restoration of nerve function. High dose corticosteroids administered systemically have a similar effect, resulting in a "medical decompression". The aim of this study is to assess the role of timing of surgery and pre operative visual status in the prognosis of direct TON undergoing EOND. Current literature lacks a proper grading system for evaluating post operative visual improvement. Hence, we have even developed a quantitative assessment for improvement of vision following EOND.

\section{MATERIALS AND METHODS}

During the last 10 years we had 31 patients with traumatic optic neuropathy - direct type; of which one patient had bilateral direct type traumatic optic neuropathy. So totally we had operated on 32 eyes. All the patients in this study were males.

\subsection{Preoperative evaluation}

Patients with traumatic optic neuropathy were admitted under neurosurgery department and references to ENT department were sent at least 24 hours after admission. However, most of these cases might already be started on high dose corticosteroids. Patients were planned for optic nerve decompression when there is radiological evidence of fracture involving optic canal (Fig. 1) and failed medical management. Patients with radiological evidence of fracture segment impingement on optic nerve and deteriorating vision were straight away taken up for EOND and not waited for medical decompression.

Ophthalmological evaluation for visual assessment (Table I) ${ }^{10}$ and fundoscopy were done for these patients. Fundoscopic examination may provide helpful information and avoid unnecessary surgery. If this examination displays total atrophy of the optic nerve (which indicate a pre existing pathology), there is no reason for EOND, because restoration of vision is impossible. Complete atrophy occurs 2 to 3 weeks after trauma. However, injuries to the intraocular or intraorbital portions of the optic nerve result in changes that may present very rapidly. Fundoscopic examination also is very helpful in ruling out etiologies of decreased vision other than injury to the optic nerve, such as retinal edema, choroidal rupture, retinal detachment, or vitreous hemorrhage ${ }^{11}$. Relative afferent pupillary defect which is a sign optic neuropathy is seen in majority of these patients. All patients who were started on high dose corticosteroids were observed for any visual improvement and not waited for more than 72 hours so that further intervention may be planned as early as possible. Those with improvement were continued with medical management. But those with no improvement or with visual deterioration were given the option of surgery. Some patients refused the surgery and unfortunately some patients could not be taken right away for surgery as they had other life threatening conditions to be treated and EOND had to be prolonged for the well being of the patient. Hence such cases were taken after 72 hours once the patient became stable for surgery. Preoperative consent regarding visual improvement and prognosis were obtained after discussing the possible complications. During the initial years of our study patients who were taken up for optic nerve decompression after 7 days with no light perception did not show any improvement. Hence in the later years, such patients were excluded.

\subsection{Surgery}

Surgery was performed under general anesthesia with patient placed supine in an Anti-Trendelenburg position. After proper decongestion of nasal cavities wide middle meatal antrostomy and ethmoidectomy were done and proceeded with identification of the natural sphenoidal ostium. A wide sphenoidotomy was done with resection of anterior sphenoid wall superiorly upto the skull base, laterally up to the lamina papyracea and medially upto the nasal septum. Once inside the sinus, the critical landmarks on the sphenoidal lateral wall were identified: the optic nerve, the internal carotid artery (ICA), and between them, the optic carotid recess. The prominence of the optic canal sometimes can be seen inside the last cell of the posterior ethmoid (Onodi cell), that can be easily detected with the pre-operative CT scan of paranasal sinuses. Unlike conventional endoscopic sinus surgeries, these landmarks were not "spot on" due to distorted anatomy as a result of fracture and fracture segments. Hence, entire mucosal covering of sphenoid sinus was denuded for better visualization and evaluation. Within the sinus, areas of traumatic dehiscence of ICA canal (cavernous and clinoid segments) were looked upon. Such dehiscences were more commonly found in well pneumatised sphe- 
noid sinus. Surgery proceeds with delineation of entire lamina papyracea; which in most cases were found to be fractured. Decompression begins by removing the lamina papyracea at the midpoint between posterior wall of maxillary sinus and orbital apex. approximately $10-15 \mathrm{~mm}$ anterior to the face of the sphenoid. Special care was taken not to injure the peri-orbita and the underlying extraocular muscles; if the periorbita are injured, fat protrudes into the field of view( if it occurs. it can be reduced by bipolar cautery). After the posterior lamina papyracea has been removed, periorbita was followed posteriorly to where they converge at the orbital apex. The thick bone between posterior ethmoid and sphenoid at the orbital apex is known as the optic tubercle. The Annulus of Zinn is attached to the superior, inferior and medial margins of the orbital junction with the optic canal and can be identified at the level of the optic tubercle. At this point, a micro-drill with a diamond burr, equipped with an irrigation system, was used to remove the optic tubercle, performing $180^{\circ}$ in circumference angle exposing the annulus of Zinn. Decompression was performed on entire length of optic canal within the sphenoid sinus till the point where the nerve curves from an oblique to a transverse direction in its posterior course, indicating that it was nearing the chiasmal region and well posterior to the encasing bony canal (Fig. 2). Finally, the optic nerve sheath and the annulus of Zinn are incised with a sickle knife, as these two structures may contribute to pressure on the optic nerve. This step of fenestration was done in our study only in selected cases; 1) when an intrasheath hematoma was suspected 2) when the optic nerve appears grossly edematous or an impression of bulging optic nerve was obtained after decompression 3) when there was a traumatic breach in the nerve sheath inflicted by a fracture segment and 4) lateral displacement of the bony optic canal with impingement on the optic nerve as well as cases with papillary edema and/or bleeding. The incision was performed in a longitudinal way on the upper-medial part, reducing the risk of damaging the ophthalmic artery, usually situated in the inferior-medial part of the optic nerve/canal (Fig. 3). In cases where we placed nerve sheath incisions, a mucosal graft was placed to prevent potential CSF leak. Also mucosal grafts from nasal cavity were placed over traumatic dehiscence of carotid canal; if any. Nasal packings were avoided as far as possible. But if any coexistent CSF leak was repaired, then packing was done using Surgicel(R) and AbGel(B)(C) without any contact with decompressed optic nerve.

After the surgical procedure, high-dose systemic administration of steroids was maintained every 8 hours for 24 hours. An ophthalmological examination was performed 24 hours after surgery.

Post operative improvement of vision was assessed 24 hours following surgery. Post operative visual improvement was classified as complete improvement, partial improvement and no improvement (Table II).

\section{RESULTS}

When surgical intervention was made within 72 hours, there was complete improvement in $59 \%$ cases; $25 \%$ cases had partial improvement and $16 \%$ had no improvement.

When done between 3 and 7 days, $31 \%$ had partial improvement and $69 \%$ had no improvement.

Beyond 7 days, there was $0 \%$ improvement (Table II)

We encountered 13 cases of intra operative CSF leaks all of which were traumatic and were not apparent before surgery. All these were repaired using fascial graft using tensor fascia lata at the time of surgery itself.

Nerve sheath fenestrations were done in 5 selected cases as discussed above of which 2 (40\%) patients had partial improvement of vision. There were no associated CSF leaks in cases where fenestration of sheath was done. However a mucosal graft was placed over the site of fenestration to prevent the chances of delayed CSF leaks.

Post operative bed side visual assessment were done 24 hours after surgery and daily till the patient was stable to be shifted to ophthalmology OPD for detailed evaluation. Earliest signs of improvement in vision were found 72 hours after surgery. Visual improvement had continued to occur for upto 8 weeks after surgery in our study.

\section{DISCUSSION}


Traumatic optic neuropathy that failed to recover with medical management should ideally be intervened as early as possible. Optic nerve being a part of the central nervous system is incapable of regeneration and if not "rescued" at the earliest, it can result in irreversible blindness. The influence of the interval between trauma and surgery is controversial. Theoretically, improvement in vision following surgery would be better when patient receives surgery as early as possible (within 72 hours). However, in a study, Dhaliwal et al. found vision improvement of $57 \%$ in patients receiving surgery within 3 days post-trauma, $58 \%$ in patients who underwent surgery between 3 and 7 days after the trauma, and $51 \%$ in the group who underwent surgery more than 7 days after the trauma ${ }^{12}$. The authors thus concluded that timing is not a prognostic factor in vision improvement. These contradictory results could be due to an absent consensus on protocols for EOND. Secondly, in this study types and severity of injury were not discussed. According to pathophysiological principles, increased pressure on the optic nerve leads to ischemia and to deterioration in visual acuity that might be irreversible after $24-48$ hours $^{13,14}$.

The possible complications of optic nerve decompression are bleeding of the sphenopalatine artery, ethmoidal arteries, ophthalmic artery, internal carotid artery, injury to the optic nerve and / or chiasm, medial rectus muscle, CSF leak, pneumoencephalus or death.

There is significant heterogeneity in the literature considering visual improvement following EOND for traumatic optic neuropathy as reviewed by Wang et al, ${ }^{15}$. Visual improvement was found in $35 \%$ to $82 \%$ of the time. ${ }^{16,12,17-29}$. Rates of improvement in patients presenting with no LP range from $0 \%$ to $62 \% 17,19,23,26,29$ , whereas rates of improvement in those with partial vision loss range from $50 \%$ to $100 \%, 19,23,28,29$. In a large systematic review of traumatic ON, rates of improvement were decreased in those with No LP preoperatively ( $41 \%$ vs. $84-93 \%$ in patients with partial vision loss) ${ }^{12}$. Although improvement in vision is seen in the majority of cases, there is insufficient follow-up data to establish whether the improvements seen in these patients are clinically meaningful. There is no quantitative assessment of visual improvement following decompression in the literature. Surgery can be considered beneficial only if the patient regains useful vision. Hence we have devised a method of quantifying the visual improvement as visual improvement scale (VIS) as follows: Complete improvement, partial improvement and no improvement. A patient is said to have complete improvement if his vision improves upto $4 / 6$ or above after surgery; partial improvement if his vision improves upto $3 / 6$ and no improvement if there is no change in vision post surgery (Table I).

Finally, unlike facial nerve, optic nerve cannot be repaired. Optic nerve which develops from central nerve system as out-pouching of the diencephalon (optic stalks) is incapable of regeneration. Whereas facial nerve is a mixed nerve which develops from second pharyngeal arch. Facial nerve injuries are initially treated with decompression and if it fails various other options are available ( direct facial-facial nerve suture, facial nerve interpositional graft, hypoglossal- facial - jump- nerve anastomosis, dynamic muscle transfer and sling plasties ${ }^{30}$. But optic nerve once atrophied cannot be repaired. Hence it is always advisable to surgically intervene as early as possible to salvage the vision in clinically indicated, properly selected patients. And it is justified to state that "Time lost is vision lost".

\section{CONCLUSION}

Traumatic optic neuropathy (TON) denotes an acute injury to the optic nerve (ON) secondary to direct or indirect trauma. Direct trauma occurs usually due to penetrating injury

causing shearing or hematoma of the optic nerve. Indirect TON is usually due to blunt head trauma and is caused by increased intracanalicular pressure from the injury with vascular ischemia and interruption of neurofeedback mechanisms leading to blindness ${ }^{28,31}$. To date no standard treatment protocol has been developed regarding traumatic optic neuropathy. However, our study shows that the timing is the most important prognostic factor in traumatic optic neuropathy. Best results are obtained when decompression is done within 72 hours. Since most of these patients have associated head injury, which needs neurosurgical intervention; it is advisable to perform optic nerve decompression in the same sitting to ensure better prognosis. Degree of vascular compromise is another determining factor for visual restoration. Unfortunately we do not still have a tool to assess the degree of optic nerve ischemia. 
Also proper selection of patient for decompression plays a vital role. The overall condition/ prognosis of the patient as well as the extend of head injury of the patient has to be considered before doing a "heroic" attempt to restore the vision. Our study did not show any added benefits with nerve sheath incision routinely. Also pre operative visual status have important role in the post operative improvement. Patient with light perception and above seems to benefit more from the surgery than patients with no perception of light.

\section{REFERENCES}

1. Ropposch T, Steger B, Meco C, et al. The effect of steroids in combination with optic nerve decompression surgery in traumatic optic neuropathy. Laryngoscope . 2013;123:1082-1086.

2. Yu-Wai-Man P, Griffiths PG. Steroids for traumatic optic neuropathy.Cochrane Database Syst Rev . 2007 ; (4):CD006032.

3. Luxenberger W, Stammberger H, Jebeles JA, Walch C. Endoscopic optic nerve decompression: the Graz experience. Laryngoscope . 1998; 108:873-882.

4. R. L. Anderson, W. R. Panje, and C. E. Gross, "Optic nerve blindness following blunt forehead trauma," Ophthalmology, vol. 89, no. 5, pp. 445-455, 1982.

5. I. Kallela, T. Hyrkas, P. Paukku, T. Iizuka, and C. Lindqvist, "Blindness after maxillofacial blunt trauma. Evaluation of candidates for optic nerve decompression surgery," Journal of

Cranio-Maxillo-Facial Surgery, vol. 22, no. 4, pp. 220-225, 1994.

6. Yu B, Ma Y, Tu Y, Wu W. The outcome of endoscopic transethmosphenoid optic canal decompression for indirect traumatic optic neuropathy with no-light-perception. J Ophthalmol.

2016;2016:6492858.

7. Yu B, Chen Y, Ma Y, Tu Y, Wu W. Outcome of endoscopic transethmosphenoid

optic canal decompression for indirect traumatic optic neuropathy in children. BMC Ophthalmol. 2018; 18(1):152. https://doi.org/10.1186/s12886-018-0792-4.

8. Tang R, Li H, Regner V, Bridges MB, Prager TC (1986) Traumatic optic neuropathy: Analysis of 37 cases. Invest Ophthalmol Vis Sci 27(suppl):102

9. Joseph MP, Lessell S, Rizzo I, Momose KI (1990) Extracranial optic nerve decompression for traumatic optic neuropathy. Arch Opthalmol 108:1091-1093

10. Kilian Schulze-Bonsel, Nicolas Feltgen, Hermann Burau, Lutz Hansen, Michael Bach; Visual Acuities "Hand Motion" and "Counting Fingers" Can Be Quantified with the Freiburg Visual Acuity Test. Invest. Ophthalmol. Vis.

11. Seiff SR. Trauma and the optic nerve. Ophthalmol Clin North Am 1992;5(3):389-94.

Sci. 2006; 47(3):1236-1240. doi: https://doi.org/10.1167/iovs.05-0981.

12. Dhaliwal SS, Sowerby LJ, Rotenberg BW. Timing of endoscopic surgical decompression in traumatic optic neuropathy: a systematic review of the literature. Int Forum Allergy Rhinol . 2016; 6:661-667.

13. Mabuchi F, Aihara M, Mackey MR, Lindsey JD, Weinreb RN. Optic nerve damage in experimental mouse ocular hypertension. Invest Ophthalmol Vis Sci. 2003;44:4321-30.

14. Levin LA. Intrinsic survival mechanisms for retinal ganglion cells. Eur J Ophthalmol. 1999; 9(Suppl. I): $12-6$.

15. Wang EW, Zanation AM, Gardner PA, et al. ICAR: endoscopic skull-base surgery. Int Forum Allergy Rhinol . 2019;9:S145-S365. https://doi.org/10.1002/alr.22326 
16. LuxenbergerW, Stammberger H, Jebeles JA,Walch C. Endoscopic optic nerve decompression: the Graz experience. Laryngoscope. 1998; 108:873-82

17. Wang DH, Zheng CQ, Qian J, Barr JJ, Anderson AG Jr. Endoscopic optic nerve decompression for the treatment of traumatic optic nerve neuropathy. ORL J Otorhinolaryngol Relat Spec . 2008; 70:130-133.

18. Rajiniganth MG, Gupta AK, Gupta A, Bapuraj JR. Traumatic optic neuropathy: visual outcome following combined therapy protocol. Arch Otolaryngol Head Neck Surg . 2003;129:1203-1206.

19. Jiang RS, Hsu CY, Shen BH. Endoscopic optic nerve decompression for the treatment of traumatic optic neuropathy. Rhinology . 2001; 39:71-74.

20. Kountakis SE, Maillard AA, El-Harazi SM, Longhini L, Urso RG. Endoscopic optic nerve decompression for traumatic blindness. Otolaryngol Head Neck Surg . 2000;123(1 Pt 1):34-37.

21. Nayak SR, Kirtane MV, Ingle MV. Transethmoid decompression of the optic nerve in head injuries: an update. J Laryngol Otol . 1991;105:205-206.

22. Emanuelli E, Bignami M, Digilio E, Fusetti S, Volo T, Castelnuovo P. Post-traumatic optic neuropathy: our surgical and medical protocol.Eur Arch Otorhinolaryngol . 2015; 272:3301-3309.

23. Hameed AS, Nampoothiri PM, Joseph B. A prospective study on outcome of endoscopic optic nerve decompression for visual loss after cranioorbital trauma. Journal of Evidence Based Medicineand Healthcare . 2014; 1:696-702.

24. Xu R, Chen F, Zuo K, et al. Endoscopic optic nerve decompression for patients with traumatic optic $.1002 /$ alr.22326 neuropathy: is nerve sheath incision necessary?

ORL J Otorhinolaryngol Relat Spec . 2014; 76:44-49.

25. Song Y, Li H, Ma Y, et al. Analysis of prognostic factors of endoscopic optic nerve decompression in traumatic blindness. Acta Otolaryngol . 2013;133:1196-1200.

26. Yang QT, Zhang GH, Liu X, Ye J, Li Y. The therapeutic efficacy of endoscopic optic nerve decompression and its effects on the prognoses of 96 cases of traumatic optic neuropathy. J Trauma Acute Care Surg . 2012; 72:1350-1355.

27. Peng A, Li Y, Hu P, Wang Q. Endoscopic optic nerve decompression for traumatic optic neuropathy in children. Int J Pediatr Otorhinolaryngol . 2011; 75:992-998.

28. Thaker A, Tandon DA, Mahapatra AK. Surgery for optic nerve injury: should nerve sheath incision supplement osseous decompression? Skull Base . 2009; 19:263-271.

29. Li H, Zhou B, Shi J, Cheng L, Wen W, Xu G. Treatment of traumatic optic neuropathy: our experience of endoscopic optic nerve decompression. J Laryngol Otol . 2008;122:1325-1329.

30. Volk GF, Pantel M, Guntinas-Lichius O. Modern concepts in facial nerve reconstruction. Head Face Med . 2010; 6:25. Published 2010 Nov 1. doi: 10.1186/1746-160X-6-25

31. Steinsapir KD,Goldberg RA. Traumatic optic neuropathy: an evolving understanding. Am J Ophthalmol 2011; 151(06):928-933.e2 skull-base surgery. Int Forum Allergy Rhinol . 2019; 9:S145-S365.

Figure legends

Figure 1: CT scans of patient with bilateral traumatic optic neuropathy: A) Black arrow showing a fracture involving right bony optic canal in the sphenoid sinus. B) Yellow arrow showing fracture segment impinging on intraorbital portion of the left optic nerve

Figure 2: Decompressed optic nerve (Blue arrow), lateral optic carotid recess (yellow star), and exposed dura (black arrow) 
Figure 3: Nerve sheath incision (black arrow) placed horizontally along the inferomedial aspect of optic nerve, orbital apex (green arrow), annulus of Zinn (blue star)
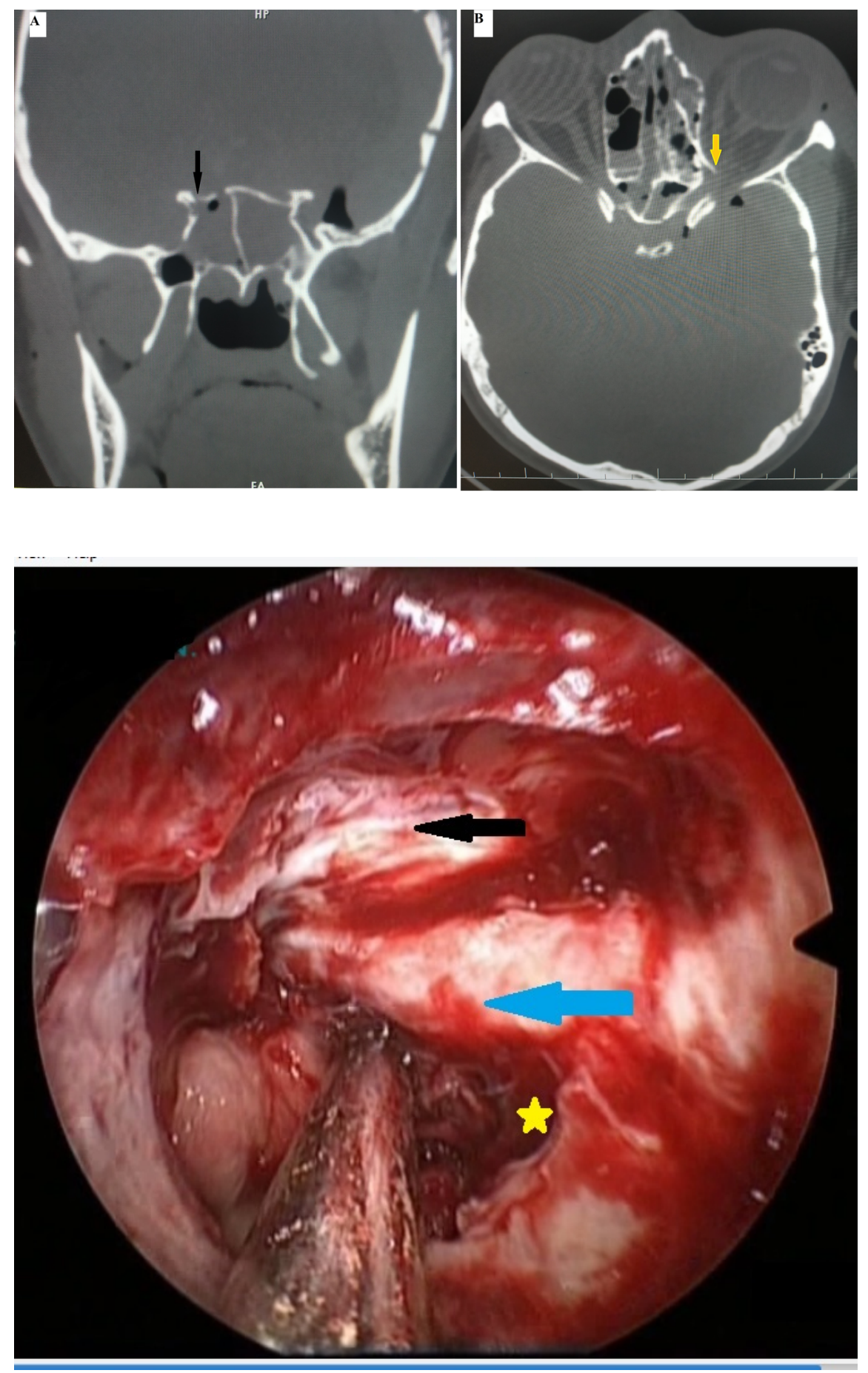


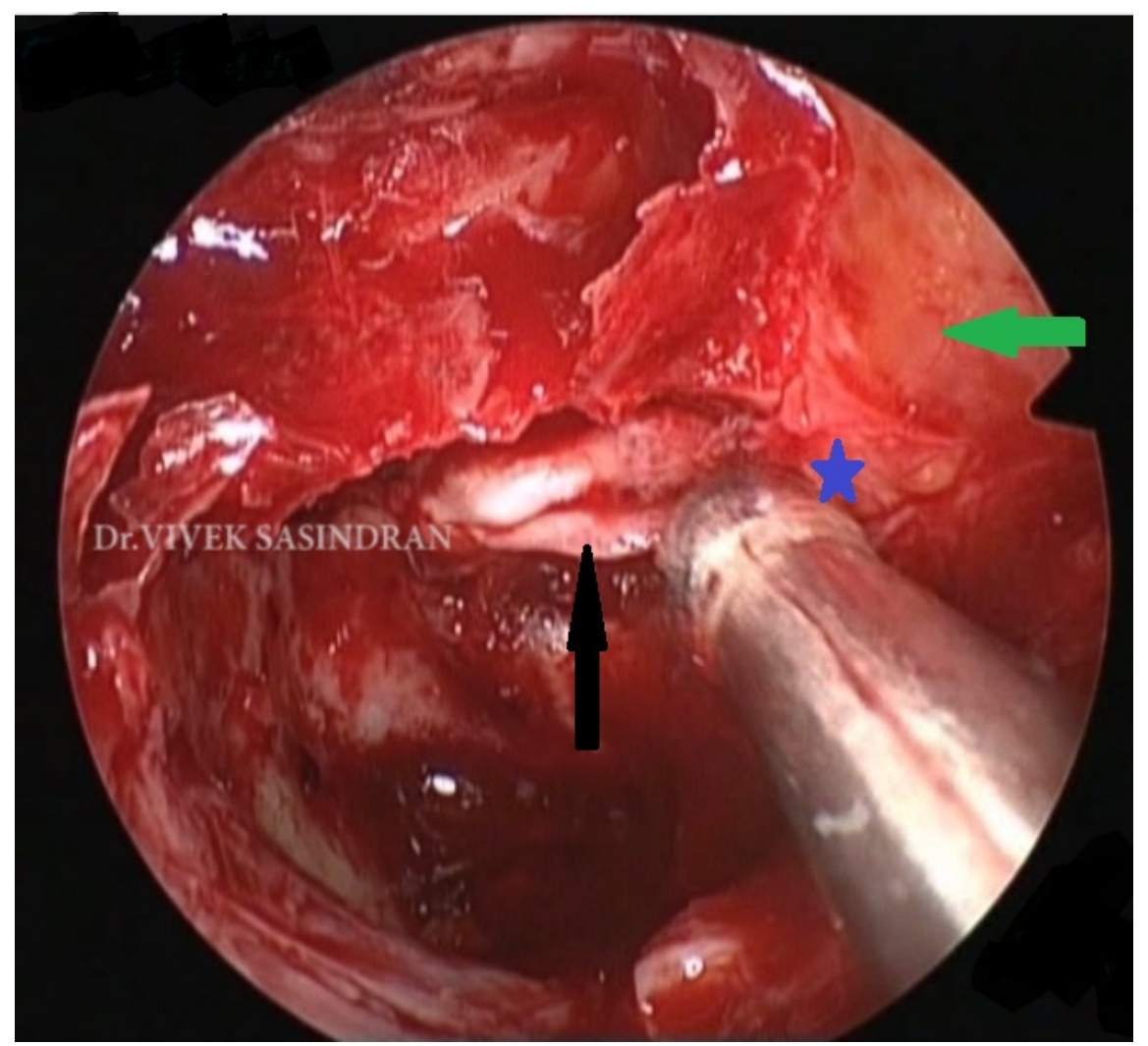

\section{Hosted file}

Tables.docx available at https://authorea.com/users/334046/articles/460113-endoscopic-opticnerve-decompression-for-direct-traumatic-optic-neuropathy-our-10-years-experience 